\title{
Discovery of basic ordinality and cardinality by young preschoolers
}

\author{
MELVIN H. MARX \\ Georgia State University, Atlanta, Georgia \\ and Florida Institute of Technology, Melbourne, Florida \\ and \\ YUNG CHE KIM \\ Keimyung University, Daegu, South Korea
}

\begin{abstract}
Fifty South Korean preschoolers were given ordinality and cardinality problems. None of the 20 children in the two younger groups (mean ages 27 and 33 months) solved either problem. All but one of the 1639 -month-olds and all 14 of the 45-month-olds solved the ordinality problem. The more difficult cardinality problem was solved by only 2 of the 39-month-olds and 9 of the 45-month-olds. There was an interaction among the variables of age, test type, and test order: Problem difficulty did not vary with test order for the 39-month-olds, but the 45-month-olds performed better on the cardinality problem when it occurred first and better on the ordinality problem when it occurred after cardinality.
\end{abstract}

Our overall purpose in this study was to compare the abilities of young preschool children at discovering the functional concepts of ordinality and cardinality, in the absence of any numerical reference. A more specific objective was to determine whether the kind of marked discontinuity in proficiency recently reported by DeLoache (1987) for the use of symbols by young preschoolers would also occur for either of the problems in the present study.

\section{METHOD}

\section{Subjects}

The subjects were all of the 2- to 4-year-old children available for testing at three day nurseries in Daegu, South Korea. A total of 50 children from four age groups were tested. Their mean ages $(S D s)$ in months were: $27.2(1.4), 32.6(1.9), 38.8(1.9)$, and $45.2(3.4)$. Both sexes were equally represented.

\section{Design}

Half of the subjects in each age group were tested first for ordinality and then shifted to cardinality testing after meeting the mastery criterion or after receiving the maximum of 10 trial blocks (100 trials) without meeting the mastery criterion. The other half of the subjects were treated in the same manner, except that the cardinality testing came first.

\section{Procedure}

In order to measure ordinality and cardinality in the absence of the usual numerical referents, it was necessary to devise new testing methods. In doing this, we were guided by the need to keep the concepts at as elementary a level as possible. The most elementary implementation

An earlier version of this report was presented at the meeting of the Psychonomic Society in Atlanta, GA in November, 1989. We thank the staff and the student participants of the day-care centers used in this study. Correspondence may be addressed to Melvin H. Marx, Department of Psychology, Florida Institute of Technology, 150 West University Blvd., Melbourne, FL 32901-6988. of ordinality seemed to be simple linear progression. Ordinality in this study was therefore defined by the movement of a hidden incentive from one receptacle to the next in line over trials. A small piece of candy, similar to an $M \& M$, was placed under one inverted cardboard cup in a linear array of five cups. The subject was instructed to turn one of the inverted cups over on each trial in order to try to obtain the incentive. If successful, he or she was allowed to eat the incentive or to keep it for later; if not, the tester indicated the correct cup by turning it over but did not give the incentive to the subject.

Cardinality was tested by putting the same reward and incentiveindication procedure in a setting in which five clusters of cups, ranging from one through five cups each, were used. The five clusters were randomly rearranged in a row on each trial, but the incentive was placed under clusters of regularly increasing size-that is, under the single cup on the first trial, the two-cup cluster on the next trial, and so forth.

Three-minute rest intervals separated trial blocks, with 10 trials to a block. The shift to the second task was marked by a change in the color of the rectangular base upon which the inverted cups were placed (from yellow to green) and of the cups themselves (from white to blue). The experimenter called attention to these changes as well as the new cup format and said that the candy was still to be found under the correct cup(s), with only one choice of cup allowed on each trial.

Testing was carried out by graduate assistants (three men, one woman) in the Department of Psychology of Keimyung University, in the city of Daegu. They had several years of experience past the Master's degree and were supervised by the second author.

\section{RESULTS}

The percentages of children solving each problem in the four age groups are shown in Figure 1. As this figure indicates, the results were clear for the 927 -month-old and the 1133 -month-old children: None solved either the ordinality or the cardinality problem. The procedures were difficult to communicate to these very young preschoolers. They were uncertain as to what to do, and picked the cups up apparently at random, often more than one at a time. As a result, no usable data were generated by these subjects. 


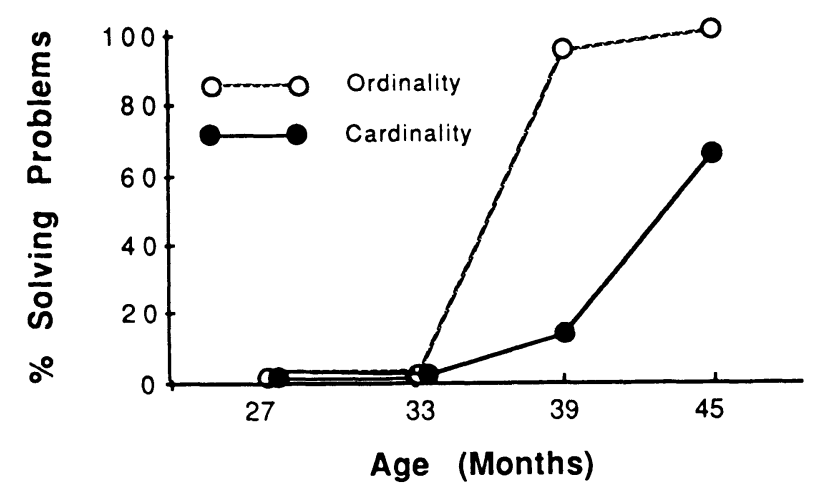

Figure 1. Mean percentage of subjects solving ordinality and cardinality problems, as a function of age.

The results for the 39- and 45-month-old children were quite different. The ordinality problem was solved by 15 of 16 of the former and all 14 of the latter. Cardinality was more difficult; only 2 of the younger group and 9 of the older group were successful.

In order to equate $n \mathrm{~s}$ (at 12) for these two groups, 4 of the younger and 2 of the older children were randomly eliminated, with little change in age means or performance means. Substantial, statistically reliable differences were found by analysis of variance in both number of trial blocks to mastery and total errors. With the latter measure, for example, the 39-month-olds made more than twice as many errors as did the 45-month-olds on the ordinality task (means of 68.5 and 29.8) and more than four times as many errors on the cardinality task (means of 24.2 and 5.5).

Statistically, overall, there were reliable effects for age [ 46.3 mean errors by the younger children, 22.7 by the older; $F(1,47)=28.28, p<.01]$ and task [54.1 mean errors on cardinality, 14.8 on ordinality; $F(1,47)=$ $170.24, p<.01$ ] but not sex [ 34.9 mean errors by males, 34.1 by females; $F(1,47)=.02, p>.05]$.

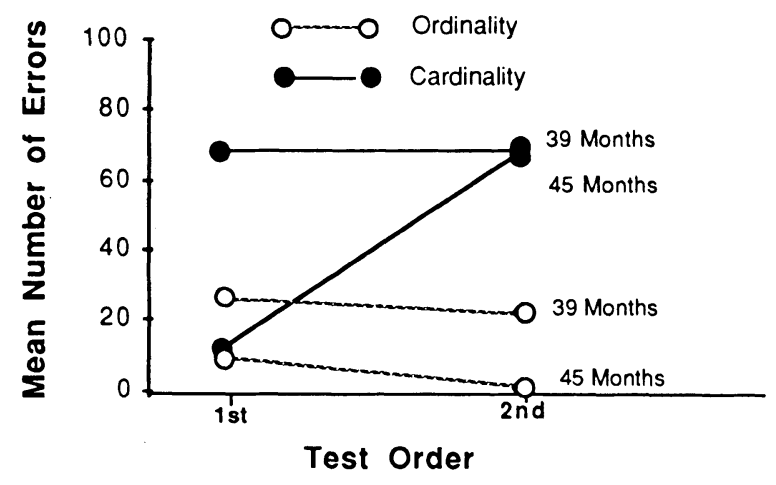

Figure 2. Mean number of errors made by 39- and 45-month-old subjects on ordinality and cardinality problems, as a function of task order.
Some surprising results occurred with the test-order variable. Overall, first tasks produced a mean of $29.1 \mathrm{er}-$ rors, second tasks a mean of 39.9 errors $[F(1,47)=8.02$, $p<.05]$. More interestingly, there was a reliable interaction between the age and test-order variables $[F(1,47)$ $=11.42, p<.01$ ]: The younger children made approximately the same number of errors on both tasks (means of 47.3 and 45.2 for first and second tasks, respectively), whereas the older children performed considerably better on the first (10.8 errors) than on the second ( 34.5 errors).

The relationships among the age, test-order, and task variables are shown in Figure 2. The indicated interaction was reliable $[F(1,47)=15.48, p<.01]$. It seems clear that the source of this interaction was the differential effect of test order on the cardinality task that occurred in the performance of the older children; apparently working first on the ordinality problem had a strong negative transfer effect on the cardinality task for these subjects, and the opposite effect occurred for the ordinality task (that is, it was easier for them when it followed cardinality).

\section{DISCUSSION}

Two major conclusions may be drawn from the results. First, with the way in which these concepts were implemented, ordinality was discovered much more readily than was cardinality. A similar conclusion was reached much earlier by Brainerd (1974), using more typical, numerical procedures, and it has been confirmed in most of the subsequent studies on the problem (e.g., Kingma \& Koops, 1981; Voight $\&$ Weiner, 1981). Moreover, we might expect an even greater difference if the ordinality component, the regular one-cup increment in correct cluster size over trials, were removed from the cardinality task.

Second, the present results indicate a precipitous development of the ability to "discover" ordinality. DeLoache's (1987) similar results on symbol use also indicate a marked discontinuity over the same age span. Although the mean age difference in the present study (32.6 to 38.8 months) was slightly less than that in DeLoache's study ( 31 to 38 months), our children showed an even greater increment (from $0 \%$ to $94 \%$ correct solutions) than that reported by DeLoache (approximately $15 \%$ to $85 \%$ correct solutions).

Our confidence in the generality of the present ordinality data needs to be tempered by the apparent difficulty the two younger groups of children had in understanding and attempting to work on the problems. How much improvement in their performance can be produced by intensified efforts to increase their comprehension of the tasks cannot, of course, be said. Also, massing of the trial blocks on both problems on the same day probably caused some hardship for the younger children. Nevertheless, the fact remains that these young preschoolers were given adequate opportunity to work on what seem to have been relatively simple tasks, and it is probable that at least a substantial part if not all of their complete failure can be attributed to cognitive inadequacy.

The interaction of task order and age is an especially interesting result, offering a fertile field for speculation. Apparently some aspect of the ordinality task interfered with subsequent cardinality performance in the older but not the younger of the two groups that provided usable data. On the one hand, it is possible that the older children were more distracted by the randomness of the positioning of the different-sized cup clusters after experiencing the perfectly ordered progression of the incentive location in the ordinality task. On the other hand, their superior performance on the ordinality task when it was given second might well be attributable to their prior experience with the ordinality inherent in the cardinality task. These strictly ad hoc suppositions cannot, 
of course, be addressed by the present data but do offer suggestions for future research.

\section{REFERENCES}

BRAINERD, C. J. (1974). Inducing ordinal and cardinal representations of the first five natural numbers. Journal of Experimental Child Psychology, 18, 520-534.

DeLoache, J. S. (1987). Rapid change in the symbolic functioning of very young children. Science, 238, 1556-1557.
KINGMA, J., \& Koops, W. (1981). On the sequentiality of ordinality and cardinality. International Journal of Behavioral Development, 4, 391-402.

Voight, F., \& Weiner, F. E. (1981). Preschool children's understanding of number: Evaluating alternative theories. German Journal of Psychology, 5, 85-88.

(Manuscript received April 2, 1990.) 